EUROPEAN JOURNAL OF PURE AND APPLIED MATHEMATICS

Vol. 13, No. 3, 2020, 529-548

ISSN 1307-5543 - www.ejpam.com

Published by New York Business Global

\title{
More on Perfect Roman Domination in Graphs
}

\author{
Leonard Mijares Paleta ${ }^{1, *}$, Ferdinand P. Jamil ${ }^{2}$ \\ 1 Department of Mathematics, College of Science and Mathematics, University of Southern \\ Mindanao, Kabacan 9407, North Cotabato, Philippines \\ 2 Department of Mathematics and Statistics, College of Science and Mathematics. \\ Center for Graph Theory, Algebra and Analysis, Premier Research of Institute of Science \\ and Mathematics, Mindanao State University-Iligan Institute of Technology, 9200 Iligan \\ City, Philippines
}

\begin{abstract}
A perfect Roman dominating function on a graph $G=(V(G), E(G))$ is a function $f: V(G) \rightarrow\{0,1,2\}$ for which each $u \in V(G)$ with $f(u)=0$ is adjacent to exactly one vertex $v \in V(G)$ with $f(v)=2$. The weight of a perfect Roman dominating function $f$ is the value $\omega_{G}(f)=\sum_{v \in V(G)} f(v)$. The perfect Roman domination number of $G$ is the minimum weight of a perfect Roman dominating function on $G$. In this paper, we study the perfect Roman domination numbers of graphs under some binary operations.
\end{abstract}

2020 Mathematics Subject Classifications: 05C22, 05C69,05C76

Key Words and Phrases: Roman dominating function, perfect Roman dominating function, Roman domination number, perfect Roman domination number

\section{Introduction}

Throughout this paper, all graphs considered are finite, simple and undirected. Let $G=(V(G), E(G)$ be a graph. The sets $V(G)$ and $E(G)$ are the vertex set and edge set, respectively, of $G$. For $S \subseteq V(G),|S|$ is the cardinality of $S$. In particular, $|V(G)|$ is called the order of $G$. For notation and terminology not given here, see [5].

Vertices $u$ and $v$ of $G$ are neighbors if $u v \in E(G)$. The open neighborhood of $v$ refers to the set $N_{G}(v)$ consisting of all neighbors of $v$. The closed neighborhood of $v$ is the set $N_{G}[v]=N_{G}(v) \cup\{v\}$. The degree of $v$, denoted $\operatorname{deg}_{G}(v)$, refers to the value $\left|N_{G}(v)\right|$, and we define $\Delta(G)=\max \left\{\operatorname{deg}_{G}(v): v \in V(G)\right\}$. Vertex $v$ is an endvertex if $\operatorname{deg}_{G}(v)=1$, and $\operatorname{End}(G)$ is the set of all endvertices of $G$. Vertex $v$ is an isolated vertex if $\operatorname{deg}_{G}(v)=0$. We denote by $I s o(G)$ the set of all isolated vertices of $G$. For $S \subseteq V(G), N_{G}(S)=\cup_{v \in S} N_{G}(v)$, and $N_{G}[S]=S \cup N_{G}(S)$.

${ }^{*}$ Corresponding author.

DOI: https://doi.org/10.29020/nybg.ejpam.v13i3.3763

Email addresses: leonard.paleta@g.msuiit.edu.ph (L. Paleta), ferdinand.jamil@g.msuiit.edu.ph (F. Jamil) 
Let $G$ and $H$ be graphs with disjoint vertex sets. The disjoint union of $G$ and $H$ is the graph $G \cup H$ with $V(G \cup H)=V(G) \cup V(H)$ and $E(G \cup H)=E(G) \cup E(H)$. The join of $G$ and $H$ is the graph $G+H$ with vertex set $V(G) \cup V(H)$ and edge set $E(G) \cup E(H) \cup\{u v$ : $u \in V(G), v \in V(H)\}$. The corona of $G$ and $H$ is the graph $G \circ H$ obtained by taking one copy of $G$ and $|V(G)|$ copies of $H$, and then joining the $i^{\text {th }}$ vertex of $G$ to every vertex in the $i^{\text {th }}$ copy of $H$. The edge corona of $G$ and $H$ is the graph $G \diamond H$ obtained by taking one copy of $G$ and $|E(G)|$ copies of $H$ and joining each of the end vertices $u$ and $v$ of each edge $u v$ of $G$ to every vertex of the copy $H^{u v}$ of $H$. The composition $G[H]$ of $G$ and $H$ is the graph with $V(G[H])=V(G) \times V(H)$ and $(u, v)\left(u^{\prime}, v^{\prime}\right) \in E(G[H])$ if and only if either $u u^{\prime} \in E(G)$ or $u=u^{\prime}$ and $v v^{\prime} \in E(H)$. The complementary prism, denoted $G \bar{G}$, is the graph formed from the disjoint union of $G$ and its complement $\bar{G}$ by adding a perfect matching between corresponding vertices of $G$ and $\bar{G}$. For the complementary prism, $V(G \bar{G})=V(G) \cup V(\bar{G})$ and $E(G \bar{G})=E(G) \cup E(\bar{G}) \cup\{v \bar{v}: v \in V(G)\}$, where $\bar{v}$ is the vertex in $\bar{G}$ corresponding to $v \in V(G)$ in the perfect matching.

A subset $S \subseteq V(G)$ is a dominating set of $G$ if $N_{G}[S]=V(G)$. The minimum cardinality of a dominating set is the domination number of $G$, denoted by $\gamma(G)$. For more details and results on domination number, we refer to $[4,9-11,13]$. In particular, if $\gamma(G)=1$ and $N_{G}[v]=V(G)$, then $v$ is said to be a dominating vertex of $G$. In this case, $\operatorname{Dom}(G)$ denotes the set of all dominating vertices of $G$. Any dominating set of $G$ of cardinality $\gamma(G)$ is called $\gamma$-set of $G$.

A dominating set $S$ of $G$ is a perfect dominating set if for every $v \in V(G) \backslash S$, there exists exactly one $u \in S$ for which $u v \in E(G)$ [16]. The minimum cardinality of a perfect dominating set is the perfect domination number of $G$, which is denoted by $\gamma^{P}(G)$. Since perfect dominating sets are dominating sets, $\gamma(G) \leq \gamma^{P}(G)$ for any graph $G$.

A Roman dominating function on $G$ is a function $f: V(G) \rightarrow\{0,1,2\}$ satisfying the condition that for each $u \in V(G)$ for which $f(u)=0$, there exists $v \in V(G)$ such that $f(v)=2$ and $u v \in E(G)$. The weight of $f$ is the value $\omega_{G}(f)=\sum_{v \in V(G)} f(v)$. The Roman domination number of $G$, denoted by $\gamma_{R}(G)$, is the minimum weight of a function $f$ on $G$. We refer to $[2,3,7,8,12,17,18]$ for the history, introduction, importance and for some of the recent developments of the study of Roman domination in graphs.

Customarily, we write $f=\left(V_{0}, V_{1}, V_{2}\right)$ for a Roman dominating function $f$ on $G$, where $V_{k}=\{v \in V(G): f(v)=k\}$. With this convention, $\omega_{G}(f)=\left|V_{1}\right|+2\left|V_{2}\right|$ and $V_{1} \cup V_{2}$ is a dominating set of $G$. In [8], it is known that for any graph $G, \gamma(G) \leq \gamma_{R}(G) \leq 2 \gamma(G)$.

A perfect Roman dominating function (or PRD-function) on $G$ is a Roman domination function $f=\left(V_{0}, V_{1}, V_{2}\right)$ on $G$ such that for each $u \in V_{0}$ there exists exactly one $v \in V_{2}$ for which $u v \in E(G)$. In other words, a $P R D$-function on $G$ is a colouring of the vertices of $G$ using colours 0,1 and 2 such that each vertex coloured 0 is adjacent to exactly one vertex coloured 2. The perfect Roman domination number of $G$, denoted by $\gamma_{R}^{P}(G)$, is the minimum weight of a $P R D$-function on $G$. A $P R D$-function $f$ with $\omega_{G}(f)=\gamma_{R}^{P}(G)$ is called $\gamma_{R}^{P}$-function of $G$.

The perfect Roman domination, a variation of the Roman domination, was introduced and first investigated in 2018 by Henning et al. [15], particularly in trees. It is further studied in [14] for regular graphs. More recent studies on the concept include $[1,19,20]$. 
In this present paper, we continue the study of perfect Roman domination, specifically on the join, corona, complementary prism, edge corona and composition of graphs.

The following bounds are established in the referred articles above.

Theorem 1.1. (i)[15] If $T$ is a tree of order $n \geq 3$, then $\gamma_{R}^{P}(T) \leq \frac{4}{5} n$;

(ii) [14] If $G$ is a k-regular graph of order $n$ with $k \geq 4$, then $\gamma_{R}^{P}(G) \leq\left(\frac{k^{2}+k+3}{k^{2}+3 k+1}\right) n$;

(iii) [19] If $G$ is a graph of order $n$, then $\gamma_{R}^{P}(G) \leq n+1-\Delta(G)$.

(iv) [19] For paths $P_{n}$ and cycles $C_{n}$ on $n \geq 3$ vertices, $\gamma_{R}^{P}\left(P_{n}\right)=\gamma_{R}^{P}\left(C_{n}\right)=\left\lceil\frac{2 n}{3}\right\rceil$.

For convenience, we adapt the symbol $P R D(G)$ to denote the set of all perfect Roman dominating functions on the graph $G$.

\section{Results}

The following proposition plays an important role in proving the desired results.

Proposition 2.1. If $f=\left(V_{0}, V_{1}, V_{2}\right)$ is a $\gamma_{R}^{P}$-function of $G$, then $\left|N_{G}(v) \cap V_{2}\right| \neq 1$ for each $v \in V_{1}$.

Proof: Suppose that there exists $v \in V_{1}$ for which $\left|N_{G}(v) \cap V_{2}\right|=1$. Consider, in particular, the function $f^{*}=\left(V_{0}^{*}, V_{1}^{*}, V_{2}^{*}\right)$ given by $f^{*}(v)=0$ and $f^{*}(x)=f(x)$ for all $x \neq v$. We have $f^{*} \in P R D(G)$ with $V_{0}^{*}=V_{0} \cup\{v\}, V_{1}^{*}=V_{1} \backslash\{v\}$ and $V_{2}^{*}=V_{2}$. Thus, $\omega_{G}\left(f^{*}\right)=\gamma_{R}^{P}(G)-1$, a contradiction.

Proposition 2.2. For a nontrivial connected graph $G$ of order $n$,

$$
\max \{2, \gamma(G)\} \leq \gamma_{R}^{P}(G) \leq \min \left\{n+1-\Delta(G), 2 \gamma^{P}(G)\right\} .
$$

Proof: Since a perfect Roman domination is a Roman domination, $\gamma(G) \leq \gamma_{R}^{P}(G)$. Let $f=\left(V_{0}, V_{1}, V_{2}\right)$ be a $\gamma_{R}^{P}$-function of $G$. If $V_{0}=\varnothing$, then $\gamma_{R}^{P}(G)=n \geq 2$. On the other hand, if $V_{0} \neq \varnothing$, then $V_{2} \neq \varnothing$ so that $\gamma_{R}^{P}(G) \geq 2\left|V_{2}\right| \geq 2$.

By Theorem 1.1(iii), $\gamma_{R}^{P}(G) \leq n+1-\Delta(G)$. Now, let $S \subseteq V(G)$ be a $\gamma^{P}$-set of $G$. Then $f=\left(V_{0}, V_{1}, V_{2}\right) \in P R D(G)$, where $V_{0}=V(G) \backslash S, V_{1}=\varnothing$ and $V_{2}=S$. Therefore, $\gamma_{R}^{P}(G) \leq 2|S|=2 \gamma^{P}(G)$.

Observe that $\gamma_{R}^{P}\left(C_{k}\right)=4=k+1-\Delta\left(C_{k}\right)<2 \gamma^{P}\left(C_{k}\right)$ for $k=5$ and $\gamma_{R}^{P}\left(C_{3 n}\right)=2 n=$ $2 \gamma^{P}\left(C_{3 n}\right)<(3 n+1)-\Delta\left(C_{3 n}\right)$ for all $n \geq 2$. Therefore, the upper bound of the inequality in Proposition 2.2 is sharp and may be determined by exactly one of $n+1-\Delta(G)$ and $2 \gamma^{P}(G)$. The inequality, however, can also be strict. To see this, note that $\gamma_{R}^{P}\left(C_{7}\right)=5<$ $\min \left\{(7+1)-\Delta\left(C_{7}\right), 2 \gamma^{P}\left(C_{7}\right)\right\}$.

Corollary 2.3. Let $G$ be a connected graph of order $n \geq 2$. Then

(i) $[19] \gamma_{R}^{P}(G)=2$ if and only if $\gamma(G)=1$. 
(ii) $\gamma_{R}^{P}(G)=n$ if and only if $n=2$.

(iii) $[19] \gamma_{R}^{P}(G)=3$ if and only if $\Delta(G)=n-2$.

(iv) If $G$ is the complete multipartite graph $K_{r_{1}, r_{2}, \ldots, r_{m}}$, where $2 \leq r_{1} \leq r_{2} \leq \ldots \leq r_{m}$, then

$$
\gamma_{R}^{P}(G)= \begin{cases}\min \left\{r_{1}+1,4\right\}, & \text { if } m=2 \\ r_{1}+1, & \text { if } m \geq 3\end{cases}
$$

Proof: Clearly, if $\gamma(G)=1$, then $\gamma^{P}(G)=1$ and the inequalities in Proposition 2.2 imply that $\gamma_{R}^{P}(G)=2$. Now, suppose that $\gamma_{R}^{P}(G)=2$, and let $f=\left(V_{0}, V_{1}, V_{2}\right)$ be a $\gamma_{R}^{P}$-function of $G$. If $V_{2}=\varnothing$, then $V(G)=V_{1}$ and $\gamma_{R}^{P}(G)=n=2$. Since $G$ is connected, $G=P_{2}$ and $\gamma(G)=1$. If $V_{2} \neq \varnothing$, then $V_{1}=\varnothing$ and $V_{2}=\{v\}$ with $N_{G}[v]=V(G)$. This means that $\gamma(G)=1$. This proves $(i)$.

If $n=2$, then $G=P_{2}$ and $\gamma_{R}^{P}(G)=2=n$. Conversely, suppose that $n \geq 3$. Pick $v \in V(G)$ such that $\operatorname{deg}_{G}(v)=\Delta(G) \geq 2$. Define on $G$

$$
f(x)= \begin{cases}2, & \text { if } x=v \\ 0, & \text { if } x \in N_{G}(v) ; \\ 1, & \text { else. }\end{cases}
$$

Then $f \in P R D(G)$ and $\omega(f)=n-(\Delta(G)-1)<n$, a contradiction. Thus, if $\gamma_{R}^{P}(G)=n$, then $n=2$. We have proved (ii).

If $\Delta(G)=n-2$, then Proposition 2.2 implies that $2 \leq \gamma_{R}^{P}(G) \leq 3$. Since $\gamma(G) \geq 2$, $\gamma_{R}^{P}(G)=3$ by $(i)$. Conversely, suppose that $\gamma_{R}^{P}(G)=3$. By $(i), \gamma(G) \geq 2$ so that $\Delta(G) \leq n-2$, and by $(i i), n \geq 4$. Let $f=\left(V_{0}, V_{1}, V_{2}\right)$ be a $\gamma_{R}^{P}$-function on $G$. If $V_{2}=\varnothing$, then $V_{1}=V(G)$ and $\gamma_{R}^{P}(G)=n \geq 4$, a contradiction. Thus, $\left|V_{2}\right|=\left|V_{1}\right|=1$, say $V_{1}=\{u\}$ and $V_{2}=\{v\}$. This means that $V(G) \backslash\{u, v\} \subseteq V_{0}$. Further, by Proposition 2.1, $u v \notin E(G)$. Accordingly, $\operatorname{deg}_{G}(v)=n-2$. Therefore, $\Delta(G) \geq n-2$. This proves (iii).

Suppose that $G$ is the complete multipartite graph described in $(i v)$. Then $\Delta(G)=$ $n-r_{1}$. Suppose first that $m=2$. Then $\gamma(G)=\gamma^{P}(G)=2$. By Proposition 2.2, $\gamma_{R}^{P}(G) \leq \min \left\{r_{1}+1,4\right\}$. Also, by $(i), \gamma_{R}^{P}(G) \geq 3$. If $r_{1}=2$, then $\gamma_{R}^{P}(G)=3=r_{1}+1$. On the other hand, if $r_{1} \geq 3$, then $\gamma_{R}^{P}(G)=4 \geq r_{1}+1$. Now, assume that $m \geq 3$. By (ii), $\gamma_{R}^{P}(G)<n$. Let $f=\left(V_{0}, V_{1}, V_{2}\right)$ be a $\gamma_{R}^{P}$-function on $G$. Then $\left|V_{2}\right|=1$, say $V_{2}=\{v\}$. Since $f$ is a $\gamma_{R}^{P}$-function, $v \in U$, where $U$ is the partite set of $G$ with $|U|=r_{1}$. More precisely, $f(v)=2, f(x)=1$ for all $x \in U \backslash\{v\}$ and $f(x)=0$ for all $x \in V(G) \backslash U$. Thus, $\gamma_{R}^{P}(G)=\omega_{G}(f)=r_{1}+1$. This proves $(i v)$.

Proposition 2.4. [19] Let $G_{1}, G_{2}, \ldots, G_{k}$ be the components of $G$. Then $\gamma_{R}^{P}(G)=$ $\sum_{j=1}^{k} \gamma_{R}^{P}\left(G_{j}\right)$.

Proposition 2.4 and Corollary 2.3(ii) yield the following corollary. 
Corollary 2.5. Let $G$ be a graph of order $n$. Then $\gamma_{R}^{P}(G)=n$ if and only if $G=\cup_{j=1}^{k} G_{j}$, where $G_{j} \in\left\{K_{1}, K_{2}\right\}$ for all $j=1,2, \ldots, k$.

Corollary 2.6. Let $G$ be a graph of order $n$. Then $\gamma(G)=\gamma_{R}^{P}(G)$ if and only if $G=\overline{K_{n}}$.

Proof: If $G=\overline{K_{n}}$, then $\gamma(G)=n$ and by Corollary $2.5, \gamma_{R}^{P}(G)=n$. Conversely, suppose that $\gamma(G)=\gamma_{R}^{P}(G)$, and let $f=\left(V_{0}, V_{1}, V_{2}\right)$ be a $\gamma_{R}^{P}$-function of $G$. Note that if $V_{2} \neq \varnothing$, then $\gamma(G) \leq\left|V_{1}\right|+\left|V_{2}\right|<\gamma_{R}^{P}(G)$, a contradiction. Thus, $V_{2}=V_{0}=\varnothing$ and $\gamma_{R}^{P}(G)=n$. This means that $\gamma(G)=n$ and, thus, $G=\overline{K_{n}}$.

\subsection{On the join of graphs}

By Corollary 2.3 $(i), \gamma_{R}^{P}\left(G+K_{n}\right)=2$ for all graphs $G$ and for all $n \geq 1$.

The following theorem characterizes all $P R D$-functions on the join of nontrivial connected graphs.

Theorem 2.7. Let $G$ and $H$ be any nontrivial connected graphs and $f=\left(V_{0}, V_{1}, V_{2}\right)$. Then $f \in P R D(G+H)$ if and only if one of the following holds:

(i) $V_{2} \subseteq V(G)$ and one of the following holds:

(a) $V_{0} \subseteq V(G), V(H) \subseteq V_{1}$ and $\left(V_{0}, V_{1} \cap V(G), V_{2}\right) \in P R D(G)$;

(b) $V_{0} \cap V(H) \neq \varnothing$ and $V_{2}=\{v\}$ for which $V_{0} \cap V(G) \subseteq N_{G}(v)$.

(ii) $V_{2} \subseteq V(H)$ and one of the following holds:

(a) $V_{0} \subseteq V(H), V(G) \subseteq V_{1}$ and $\left(V_{0}, V_{1} \cap V(H), V_{2}\right) \in P R D(H)$;

(b) $V_{0} \cap V(G) \neq \varnothing$ and $V_{2}=\{v\}$ for which $V_{0} \cap V(H) \subseteq N_{H}(v)$.

(iii) $A_{1}=V_{2} \cap V(G) \neq \varnothing$ and $A_{2}=V_{2} \cap V(H) \neq \varnothing$ and the following holds:

(a) If $V_{0} \cap V(G) \neq \varnothing$, then $\left|A_{2}\right|=1$ and $\left(V_{0} \cap V(G)\right) \cap N_{G}\left(A_{1}\right)=\varnothing$;

(b) If $V_{0} \cap V(H) \neq \varnothing$, then $\left|A_{1}\right|=1$ and $\left(V_{0} \cap V(H)\right) \cap N_{H}\left(A_{2}\right)=\varnothing$.

Proof: Assume that $f$ is a perfect Roman dominating function on $G+H$. We consider three cases:

Case 1: Suppose that $V_{2} \subseteq V(G)$. If $V_{0} \subseteq V(G)$, then $V(H) \subseteq V_{1}$ and the restriction $\left.f\right|_{V(G)}=\left(V_{0}, V_{1} \cap V(G), V_{2}\right)$ of $f$ on $G$ is a perfect dominating function on $G$. Suppose that $V_{0} \cap V(H) \neq \varnothing$. Then, $\left|V_{2}\right|=1$, say $V_{2}=\{v\}$. Necessarily, $V_{0} \cap V(G) \subseteq N_{G}(v)$.

Case 2: Similarly, if $V_{2} \subseteq V(H)$, then either $(i i)(a)$ or $(i i)(b)$ holds.

Case 3: Assume that $V_{2}$ intersects both $V(G)$ and $V(H)$, and $A_{1}=V_{2} \cap V(G)$ and $A_{2}=V_{2} \cap V(H)$. Suppose that $V_{0} \cap V(G) \neq \varnothing$, and let $v \in V_{0} \cap V(G)$. Since $A_{2} \subseteq N_{G+H}(v)$, $\left|A_{2}\right|=1$ and $v \notin N_{G}\left(A_{1}\right)$. Since $v$ is arbitrary, (iii)(a) holds. Similarly, (iii)(b) holds.

Conversely, suppose that $(i)(a)$ holds for $f$, and let $w \in V_{0}$. Then $w \in V(G)$ and there exists a unique $u \in V_{2}$ for which $u w \in E(G)$. Since $V(H) \subseteq V_{1}, u$ is unique in $V(G+H)$ for 
which $u w \in E(G+H)$. This means that $f \in P R D(G+H)$. Suppose that $(i)(b)$ holds for $f$, and let $w \in V_{0}$. Whether $w \in V(G)$ or $w \in V(H), v$ is a unique element in $V_{2}$ for which $w v \in E(G+H)$. Thus, $f \in P R D(G+H)$. Similarly, if (ii) holds, the same conclusion is attained for $f$. Suppose now that (iii) holds for $f$. Let $v \in V_{0}$. If $v \in V(G)$, then by condition $(a), A_{2}=\{u\}$ for some $u \in V(H)$ and $N_{G+H}(v)=\{u\}$. Similarly, if $v \in V(H)$, then $A_{1}=\{u\}$ for some $u \in V(G)$ and $N_{G+H}(v)=\{u\}$. Accordingly, $f \in P R D(G+H)$.

We now use Theorem 2.7 to prove the following result which is also provided in [19].

Corollary 2.8. [19] Let Gand $H$ be nontrivial connected graphs of orders $m$ and $n$, respectively. Then

$$
\gamma_{R}^{P}(G+H)=\min \{4+\delta(G)+\delta(H), m+1-\Delta(G), n+1-\Delta(H)\} .
$$

Proof: Let $\alpha=\min \{4+\delta(G)+\delta(H), m+1-\Delta(G), n+1-\Delta(H)\}$. Let $v \in V(G)$ for which $\operatorname{deg}_{G}(v)=\Delta(G)$. Define $f=\left(V_{0}, V_{1}, V_{2}\right)$ on $G+H$ by

$$
f(x)= \begin{cases}2, & \text { if } x=v ; \\ 0, & \text { if } x \in V(H) \cup N_{G}(v) ; \\ 1, & \text { else. }\end{cases}
$$

Since $f$ satisfies condition $(i)(b)$ of Proposition 2.7, $f=\left(V_{0}, V_{1}, V_{2}\right) \in P R D(G+H)$ with $V_{2}=\{v\}$ and $V_{1}=V(G) \backslash N_{G}[v]$. Thus,

$$
\begin{aligned}
\gamma_{R}^{P}(G+H) \leq \omega_{G+H}(f) & =\left|V(G) \backslash N_{G}[v]\right|+2 \\
& =m+1-\Delta(G) .
\end{aligned}
$$

Similarly, $\gamma_{R}^{P}(G+H) \leq n+1-\Delta(H)$.

Now, pick $u \in V(G)$ and $v \in V(H)$ such that $\operatorname{deg}_{G}(u)=\delta(G)$ and $\operatorname{deg}_{H}(v)=\delta(H)$, and define $f=\left(V_{0}, V_{1}, V_{2}\right)$ on $G+H$ by

$$
f(x)= \begin{cases}2, & \text { if } x=u, v \\ 1, & \text { if } x \in N_{G}(u) \cup N_{H}(v) ; \\ 0, & \text { else. }\end{cases}
$$

Since $f$ satisfies Proposition $2.7(i i i), f \in P R D(G+H)$. Since $V_{2}=\{u, v\}$ and $V_{1}=$ $N_{G}(u) \cup N_{H}(v)$,

$$
\begin{aligned}
\gamma_{R}^{P}(G+H) \leq \omega_{G+H}(f) & =\left|N_{G}(u) \cup N_{H}(v)\right|+4 \\
& =4+\delta(G)+\delta(H) .
\end{aligned}
$$

All of the above show that $\gamma_{R}^{P}(G+H) \leq \alpha$.

Now, let $f=\left(V_{0}, V_{1}, V_{2}\right)$ be a $\gamma_{R}^{P}$-function of $G+H$. By Corollary 2.3(ii), since $m+n \geq 4, V_{2} \neq \varnothing$. Assume $A_{1}=V_{2} \cap V(G) \neq \varnothing$. We consider two cases: 
Case 1: Suppose that $A_{2}=V_{2} \cap V(H)=\varnothing$. If Proposition 2.7 $(i)(a)$ holds for $f$, then

$$
\omega_{G+H}(f) \geq n+\gamma_{R}^{P}(G)>n \geq n+1-\Delta(H) \geq \alpha .
$$

On the other hand, if Proposition 2.7(i)(b) holds for $f$, then

$$
\omega_{G+H}(f) \geq 2+\left|V(G) \backslash N_{G}[v]\right| \geq m+1-\Delta(G) \geq \alpha .
$$

Case 2: Suppose that $A_{2}=V_{2} \cap V(H) \neq \varnothing$. If $\left|A_{1}\right| \geq 2$ and $\left|A_{2}\right| \geq 2$, then $V_{0}=\varnothing$ and $\gamma_{R}^{P}(G+H)>m+n$, which is impossible. Assume that $\left|A_{2}\right|=1$. We consider two subcases. First, suppose that $\left|A_{1}\right| \geq 2$. Then $V_{0} \cap V(H)=\varnothing$, and since $f$ is a $\gamma_{R}^{P}$-function of $G+H, V(G) \backslash N_{G}\left[A_{1}\right] \subseteq V_{0}$ (by Proposition 2.1) and $N_{G}\left(A_{1}\right) \backslash A_{1} \subseteq V_{1}$. This means that $\left|V_{1}\right| \geq\left|V(H) \backslash V_{2}\right|+\left|N_{G}\left(A_{1}\right) \backslash A_{1}\right|$ so that

$$
\omega_{G+H}(f)=(n-1)+\left|N_{G}\left(A_{1}\right) \backslash A_{1}\right|+2\left|V_{2}\right| \geq n+5>n+1-\Delta(H) .
$$

Finally, suppose that $\left|A_{1}\right|=1$. Let $A_{1}=\{u\}$ and $A_{2}=\{v\}$ for some $u \in V(G)$ and $v \in V(H)$. By Proposition 2.1, $f(x)=0$ for all $x \in V(G+H) \backslash\left(N_{G}[u] \cup N_{H}[v]\right)$. Thus,

$$
\omega_{G+H}(f) \geq 2\left|A_{1} \cup A_{2}\right|+\left|N_{G}(u) \cup N_{H}(v)\right| \geq 4+\delta(G)+\delta(H) \geq \alpha .
$$

All cases above imply that $\gamma_{R}^{P}(G+H) \geq \alpha$.

In particular, if $m \geq n$, then

$$
\gamma_{R}^{P}\left(P_{m}+P_{n}\right)=\left\{\begin{array}{ll}
n-1, & \text { if } n \leq 6 ; \\
6, & \text { if } n \geq 7 .
\end{array} \text { and } \gamma_{R}^{P}\left(C_{m}+P_{n}\right)= \begin{cases}n-1, & \text { if } n \leq 7 \\
7, & \text { if } n \geq 8\end{cases}\right.
$$

\subsection{On the corona of graphs}

Let $G$ and $H$ be connected graphs. Adapting the notation used in [6], for each $v \in$ $V(G), H^{v}$ denotes that copy of $H$ which is joined with $v$ in $G \circ H$. In case $H=\{x\}$, we write $V\left(H^{v}\right)=\left\{x^{v}\right\}$. Then $V(G+H)=\cup_{v \in V(G)} V\left(H^{v}+v\right)$, where $H^{v}+v=H^{v}+\langle v\rangle$.

It is worth noting that $K_{1} \circ H=H+K_{1}$ for any graph $H$.

Theorem 2.9. For nontrivial connected graphs $G$ of order n,

$$
\gamma_{R}^{P}\left(G \circ K_{1}\right)=\min \left\{\omega_{G}(f)+n-\left|V_{2}\right|: f=\left(V_{0}, V_{1}, V_{2}\right) \in P R D(G)\right\} .
$$

In particular, $\gamma_{R}^{P}\left(K_{n} \circ K_{1}\right)=n+1$.

Proof: Write $H=\{x\}$, and put $\alpha=\min \left\{\omega_{G}(f)+n-\left|V_{2}\right|: f=\left(V_{0}, V_{1}, V_{2}\right) \in P R D(G)\right\}$. Let $f=\left(V_{0}, V_{1}, V_{2}\right) \in P R D(G)$. Define $f^{*}=\left(V_{0}^{*}, V_{1}^{*}, V_{2}^{*}\right)$ on $G \circ K_{1}$ by

$$
f^{*}(z)= \begin{cases}f(z), & \text { if } z \in V(G) \\ 1, & \text { if } z=x^{v} \text { for some } v \in V_{0} \cup V_{1} \\ 0, & \text { if } z=x^{v} \text { for some } v \in V_{2}\end{cases}
$$


Then $f^{*} \in P R D\left(G \circ K_{1}\right)$ with $V_{0}^{*}=V_{0} \cup\left\{x^{v}: v \in V_{2}\right\}, V_{1}^{*}=V_{1} \cup\left\{x^{v}: v \in V_{0} \cup V_{1}\right\}$ and $V_{2}^{*}=V_{2}$. Moreover,

$$
\omega_{G \circ K_{1}}\left(f^{*}\right)=\omega_{G}(f)+n-\left|V_{2}\right| .
$$

Thus, $\gamma_{R}^{P}\left(G \circ K_{1}\right) \leq \alpha$.

Let $f=\left(V_{0}, V_{1}, V_{2}\right)$ be a $\gamma_{R}^{P}$-function on $G \circ K_{1}$, and let $A$ denote the set of all $u \in V_{0} \cap V(G)$ for which $u v \notin E(G)$ for all $v \in V_{2} \cap V(G)$. Then for each $u \in A$, $V_{2} \cap N_{G \circ K_{1}}(u)=\left\{x^{u}\right\}$. Define $f^{*}=\left(V_{0}^{*}, V_{1}^{*}, V_{2}^{*}\right)$ on $G \circ K_{1}$ by

$$
f^{*}(z)= \begin{cases}f(z), & \text { if } z \in V(G) \backslash A ; \\ 1, & \text { if } z \in A \cup\left\{x^{u}: u \in\left(V_{0} \cup V_{1}\right) \cap V(G)\right\} ; \\ 0, & \text { if } z \in\left\{x^{v}: v \in V_{2} \cap V(G)\right\} .\end{cases}
$$

Then $f^{*} \in P R D\left(G \circ K_{1}\right)$ with $V_{0}^{*}=\left(\left(V_{0} \cap V(G)\right) \backslash A\right) \cup\left\{x^{u}: u \in V_{2} \cap V(G)\right\}, V_{1}^{*}=$ $A \cup\left(V_{1} \cap V(G)\right) \cup\left\{x^{u}: u \in\left(V_{0} \cup V_{1}\right) \cap V(G)\right\}$ and $V_{2}^{*}=V_{2} \cap V(G)$. Observe that $f(u)+f\left(x^{u}\right)=2=f^{*}(u)+f^{*}\left(x^{u}\right)$ for each $u \in A$, and $f(u)+f\left(x^{u}\right) \geq f^{*}(u)+f^{*}\left(x^{u}\right)$ for each $u \in V(G) \backslash A$. Thus,

$$
\begin{aligned}
\omega_{G \circ K_{1}}(f) & =\sum_{u \in A}\left(f(u)+f\left(x^{u}\right)\right)+\sum_{v \in V(G) \backslash A}\left(f(u)+f\left(x^{u}\right)\right) \\
& \geq \sum_{u \in A}\left(f^{*}(u)+f^{*}\left(x^{u}\right)\right)+\sum_{u \in V(G) \backslash A}\left(f^{*}(u)+f^{*}\left(x^{u}\right)\right) \\
& =\omega_{G \circ K_{1}}\left(f^{*}\right) .
\end{aligned}
$$

Since $f$ is a $\gamma_{R}^{P}$-function, $\omega_{G \circ K_{1}}(f)=\omega_{G \circ K_{1}}\left(f^{*}\right)$. Moreover, for each $u \in V_{0}^{*} \cap V(G)$, $u \in\left(V_{0} \cap V(G)\right) \backslash A$ so that there exists a unique $v \in V_{2} \cap V(G)=V_{2}^{*}$ such that $u v \in E(G)$. This means that the restriction $\left.f^{*}\right|_{G}$ of $f^{*}$ to $G$ is a perfect Roman dominating function on $G$. Thus,

$$
\begin{aligned}
\gamma_{R}^{P}\left(G \circ K_{1}\right)=\omega_{G \circ K_{1}}\left(f^{*}\right) & =\omega_{G}\left(\left.f^{*}\right|_{G}\right)+\sum_{v \in V(G)} f^{*}\left(x^{v}\right) \\
& =\omega_{G}\left(\left.f^{*}\right|_{G}\right)+\left|\left(V_{0} \cup V_{1}\right) \cap V(G)\right| \\
& =\omega_{G}\left(\left.f^{*}\right|_{G}\right)+n-\left|V_{2}^{*} \cap V(G)\right| \\
& \geq \alpha .
\end{aligned}
$$

It follows from Theorem 2.9 that for all connected graphs $G$ of order $n \geq 2$,

$$
\gamma_{R}^{P}\left(G \circ K_{1}\right) \leq \gamma_{R}^{P}(G)+n-\lambda,
$$

where $\lambda=\max \left\{\left|V_{2}\right|:\left(V_{0}, V_{1}, V_{2}\right)\right.$ is a $\gamma_{R}^{P}$-function on $\left.G\right\}$, and this bound is sharp. Verify that equality is attained if $G$ is a cycle $C_{n}(n \geq 3)$, a path $P_{n}(n \geq 2)$, or any graph with $\gamma(G)=1$.

Our desired result for more general graphs $G$ and $H$ will follow from the following characterization. 
Theorem 2.10. Let $G$ and $H$ be nontrivial graphs with $G$ connected, and $f=\left(V_{0}, V_{1}, V_{2}\right)$. Then $f \in P R D(G \circ H)$ if and only if the following holds:

(i) For all $v \in V_{0} \cap V(G)$ either

(a) $V_{2} \cap N_{G}(v)=\varnothing$ and $V_{2} \cap V\left(H^{v}\right)=\{u\}$ with u satisfying $V_{0} \cap V\left(H^{v}\right) \subseteq N_{H^{v}}(u)$; or

(b) $\left|V_{2} \cap N_{G}(v)\right|=1$ and $V\left(H^{v}\right) \subseteq V_{1}$;

(ii) For all $v \in V_{1} \cap V(G)$, the restriction $\left.f\right|_{H^{v}}$ of $f$ to $H^{v}$ is a perfect Roman dominating function on $H^{v}$;

(iii) For all $v \in V_{2} \cap V(G)$ for which $V_{0} \cap V\left(H^{v}\right) \neq \varnothing, V_{0} \cap N_{H^{v}}\left(V_{2} \cap V\left(H^{v}\right)\right)=\varnothing$.

Proof: Assume that $f \in P R D(G \circ H)$. Let $v \in V_{0} \cap V(G)$. Then there exists a unique $u \in V_{2}$ for which $u \in N_{G \circ H}(v)=V\left(H^{v}\right) \cup N_{G}(v)$. If $V_{2} \cap N_{G}(v)=\varnothing$, then $V_{2} \cap V\left(H^{v}\right)=\{u\}$ and $V_{0} \cap V\left(H^{v}\right) \subseteq N_{H^{v}}(u)$. Suppose that $V_{2} \cap N_{G}(v) \neq \varnothing$. Then $\left|V_{2} \cap N_{G}(v)\right|=1$ and $V_{2} \cap V\left(H^{v}\right)=\varnothing$. Moreover, if $w \in V_{0} \cap V\left(H^{v}\right)$, then there exists a unique $z \in V_{2} \cap V\left(H^{v}\right)$ such that $w z \in E\left(H^{v}\right)$. Since $v z \in E(G \circ H)$, this is impossible. Thus, $V\left(H^{v}\right) \subseteq V_{1}$. This proves $(i)$. Next, let $v \in V_{1} \cap V(G)$, and let $w \in V_{0} \cap V\left(H^{v}\right)$. Since $f$ is a perfect Roman dominating function, there exists unique $u \in V_{2}$ for which $u w \in E(G \circ H)$. Since $v \in V_{1}$, $u \in V_{2} \cap V\left(H^{v}\right)$ and $u w \in E\left(H^{v}\right)$. Thus, $\left.f\right|_{H^{v}}$ is a perfect Roman dominating function on $H^{v}$, and $(i i)$ holds. Statement $(i i i)$ is clear.

Conversely, suppose that conditions $(i)$, (ii) and (iii) hold for $f$, and let $w \in V_{0}$. Then $w \in V\left(H^{v}+v\right)$ for some $v \in V(G)$. If $w=v$, then by condition $(i), V_{2} \cap\left(V\left(H^{v}\right) \cup N_{G}(w)\right)=$ $\{u\}$ for some $u \in V(G \circ H)$. This means that $V_{2} \cap N_{G \circ H}(w)=\{u\}$. Suppose that $w \in V\left(H^{v}\right)$. We consider three cases:

Case 1: Suppose that $v \in V_{0}$. Since $w \in V_{0} \cap V\left(H^{v}\right), V\left(H^{v}\right) \nsubseteq V_{1}$. Thus, by condition (i) there exists $u \in V\left(H^{v}\right)$ for which $V_{2} \cap V\left(H^{v}\right)=\{u\}$ and $V_{0} \cap V\left(H^{v}\right) \subseteq N_{H^{v}}(u)$. This means that $V_{2} \cap N_{G \circ H}(w)=\{u\}$.

Case 2: Suppose that $v \in V_{1}$. By condition (ii), there exists a unique $u \in V_{2} \cap V\left(H^{v}\right)$ such that $u w \in E\left(H^{v}\right) \subseteq E(G \circ H)$. This implies that $V_{2} \cap N_{G \circ H}(w)=\{u\}$.

Case 3: Suppose that $v \in V_{2}$. Since $w \in V_{0} \cap V\left(H^{v}\right)$, condition (iii) implies that $w \notin N_{H^{v}}\left(V_{2} \cap V\left(H^{v}\right)\right.$. Thus, $V_{2} \cap N_{G \circ H}(w)=\{v\}$.

Therefore, $f$ is a perfect Roman dominating function on $V(G \circ H)$.

Corollary 2.11. Let $G$ and $H$ be nontrivial graphs with $G$ connected of order $n$. Then $\gamma_{R}^{P}(G \circ H)=2 n$.

Proof: By Theorem 2.7, the function $f=\left(V_{0}, V_{1}, V_{2}\right)$ defined by $f(x)=2$ for all $v \in V(G)$, and $f(x)=0$ else, is a perfect Roman dominating function on $G \circ H$. Thus, $\gamma_{R}^{P}(G \circ H) \leq 2 n$.

Now, let $f=\left(V_{0}, V_{1}, V_{2}\right)$ be a $\gamma_{R}^{P}$-function on $V(G \circ H)$. Let $v \in V(G)$. Clearly, if $v \in V_{2}$, then $\sum_{x \in V\left(H^{v}+v\right)} f(x) \geq 2$. If $v \in V_{0}$, then by Proposition $2.10(i)$ and since 
$\mid V\left(H^{v} \mid \geq 2, \sum_{x \in V\left(H^{v}+v\right)} f(x) \geq 2\right.$. Finally, if $v \in V_{1}$, then by Proposition 2.10(ii), $\sum_{x \in V\left(H^{v}+v\right)} f(x)>2$. Therefore,

$$
\gamma_{R}^{P}(G \circ H)=\omega_{G \circ H}(f)=\sum_{v \in V(G)}\left(\sum_{x \in V\left(H^{v}+v\right)} f(x)\right) \geq 2 n .
$$

\subsection{On the complementary prisms}

Let $f=\left(V_{0}, V_{1}, V_{2}\right) \in P R D(G \bar{G})$. Suppose that for the restriction $\left.f\right|_{\bar{G}} \notin P R D(\bar{G})$. Then there exists $v \in V(G)$ such that $\bar{v} \in V_{0}$ and $V_{2} \cap N_{G \bar{G}}(\bar{v})=\{v\}$. Let $u \in V_{0} \cap V(G)$. There exists $w \in V(G \bar{G})$ such that $V_{2} \cap N_{G \bar{G}}(u)=\{w\}$. If $w=\bar{u}$, then $\overline{u v} \notin E(\bar{G}$, and consequently, $u v \in E(G)$, a contradiction. Thus, $w \in V_{2} \cap V(G)$. This proves the following lemma.

Lemma 2.12. Let $G$ be any graph. If $f \in P R D(G \bar{G})$, then $\left.f\right|_{G} \in P R D(G)$ or $\left.f\right|_{\bar{G}} \in$ $\operatorname{PRD}(\bar{G})$.

Proposition 2.13. Let $G$ be a graph of order $n$. Then

(i) $\gamma(G \bar{G})<\gamma_{R}^{P}(G \bar{G})$;

(ii) $\gamma_{R}^{P}(G \bar{G})=2$ if and only if $n=1$;

(iii) $\gamma_{R}^{P}(G \bar{G})=3$ if and only if $G \in\left\{K_{2}, \overline{K_{2}}\right\}$;

(iv) If $\gamma(G)=1$, then $\gamma_{R}^{P}(G \bar{G}) \leq n+1$ and equality is attained if $\operatorname{deg}_{G}(v) \leq 3$ for all $v \notin \operatorname{Dom}(G)$ or $\bar{G}$ is the disjoint union of $K_{j} \in\left\{K_{1}, K_{2}\right\}$.

Proof: Since $G \bar{G}$ is connected, (i) follows from Corollary 2.6.

If $n=1$, then $G \bar{G}=K_{2}$ and $\gamma_{R}^{P}(G \bar{G})=2$. Suppose that $\gamma_{R}^{P}(G \bar{G})=2$, and let $f$ be a $\gamma_{R}^{P}$-function of $G \bar{G}$. By Lemma 2.12 , we may assume that $\left.f\right|_{G} \in P R D(G)$. If $\omega_{G}\left(\left.f\right|_{G}\right)=1$, then $n=1$. If $\omega_{G}\left(\left.f\right|_{G}\right)=2$, then $G=\{v\}$ with $f(v)=\left.f\right|_{G}(v)=2$ and $f(\bar{v})=0$.

If $G \in\left\{K_{2}, \overline{K_{2}}\right\}$, then $G \bar{G}$ is isomorphic to $P_{4}$. Thus, $\gamma_{R}^{P}(G \bar{G})=3$. Conversely, suppose that $\gamma_{R}^{P}(G \bar{G})=3$. By Proposition 2.3(iii), $\Delta(G \bar{G})=2 n-2$. Let $v \in V(G \bar{G})$ be such that $\operatorname{deg}_{G \bar{G}}(v)=2 n-2$. Without loss of generality, assume that $v \in V(G)$. Since $N_{G \bar{G}}(v) \cap V(\bar{G})=\{\bar{v}\}, \operatorname{deg}_{G}(v)=2 n-3 \leq n-1$. Necessarily, $n \leq 2$. By $(i i), n=2$ and $G=K_{2}$.

If $\gamma(G)=1$, then by Proposition $2.2, \gamma_{R}^{P}(G \bar{G}) \leq n+1$. First, suppose that $\operatorname{deg}_{G}(v) \leq 3$ for all $v \notin \operatorname{Dom}(G)$. Let $f=\left(V_{0}, V_{1}, V_{2}\right)$ be a $\gamma_{R}^{P}$-function of $G \bar{G}$. Since $\omega_{G \bar{G}}(f) \leq n+1$, $V_{2} \neq \varnothing$. We consider two cases: 
Case 1: Suppose that $V_{2} \cap V(G)=\varnothing$. If $V(G) \subseteq V_{1}$, then $V(\bar{G}) \nsubseteq V_{0}$ so that $\omega_{G \bar{G}}(f) \geq$ $n+1$. Suppose that $V(G) \cap V_{0} \neq \varnothing$. Then

$$
\begin{aligned}
\omega_{G \bar{G}}(f) & =\sum_{w \in V_{0} \cap V(G)} f(\bar{w})+\sum_{w \in V_{1} \cap V(G)}(f(w)+f(\bar{w})) \\
& \geq n+1 .
\end{aligned}
$$

Case 2: Assume that $V_{2} \cap V(G) \neq \varnothing$. We consider two subcases:

Subcase 2.1: Suppose that $V_{2}$ contains a dominating vertex $v$ of $G$. Since $f$ is a $\gamma_{R}^{P}$-function, $N_{G}(v) \cup\{\bar{v}\} \subseteq V_{0}$. Let $w \in V(G) \backslash\{v\}$. Suppose that $\bar{w} \in V_{0}$. There exists $u \in V(G)$ such that $N_{\bar{G}}(\bar{w}) \cap V_{2}=\{\bar{u}\}$. Since $\overline{w v} \notin E(\bar{G}), u \neq v$. Thus, $u \in V_{0}$ and $v, \bar{u} \in N_{G \bar{G}}(u) \cap V_{2}$, a contradiction. This means that $f(\bar{w}) \geq 1$. Therefore, $\omega_{G \bar{G}}(f)=$ $2+\sum_{w \in V(G) \backslash\{v\}} f(\bar{w}) \geq 2+n-1=n+1$.

Subcase 2.2: Suppose that $V_{2} \cap \operatorname{Dom}(G)=\varnothing$. Choos $v \in \operatorname{Dom}(G)$. Put $A=\{w \in$ $V(G): f(w)=f(\bar{w})=0\}$. If $A=\varnothing$, then $f(w)+f(\bar{w}) \geq 1$ for all $w \in V(G)$ and since $V_{2} \cap V(G) \neq \varnothing$, we have $\omega_{G \bar{G}}(f) \geq n+1$. Suppose that $A \neq \varnothing$. Here, we work on two subcases:

Subcase 2.2.1: Suppose that $v \in V_{0}$. If $f(\bar{v})=2$, then $V(G) \cap V_{2}=\varnothing$ and so $f(\bar{u})=2$ for each $u \in V_{0} \cap V(G)$. This implies that $\omega_{G \bar{G}}(f) \geq n+1$. Suppose that $f(\bar{v})=1$. Then there exists $u \in V(G)$ such that $V_{2} \cap V(G)=\{u\}$. Moreover, for each $w \in A, w u \in E(G)$. Since $\operatorname{deg}_{G}(u) \leq 3$ and $u v \in E(G),|A| \leq 2$. Suppose that $A=\{w\}$. There exists $a \in V(G)$ such that $u \neq a$ and $N_{\bar{G}}(\bar{w}) \cap V_{2}=\{\bar{a}\}$. Since $\alpha=(f(u)+f(\bar{u}))+(f(w)+f(\bar{w}))+(f(a)+f(\bar{a})) \geq 4$,

$$
\omega_{G \bar{G}}(f)=\alpha+\sum_{x \in V(G) \backslash\{u, w, a\}}(f(x)+f(\bar{x})) \geq 4+(n-4)+1=n+1 .
$$

Now, suppose that $A=\{w, z\}$. There exist $a, b \in V(G)$ such that $\bar{a}, \bar{b} \in V_{2}, \overline{w a}, \bar{z} \bar{b} \in E(\bar{G})$ and $\bar{a}, \bar{b} \in N_{\bar{G}}(\bar{u})$. Thus, $f(\bar{u})=f(a)=f(b)=1$ and whether $a=b$ or $a \neq b$,

$$
\alpha=(f(u)+f(\bar{u}))+(f(w)+f(\bar{w}))+(f(z)+f(\bar{z}))+(f(a)+f(\bar{a}))+(f(b)+f(\bar{b})) \geq 6 .
$$

Thus,

$$
\omega_{G \bar{G}}(f)=\alpha+\sum_{x \in V(G) \backslash\{u, w, z, a, b\}}(f(x)+f(\bar{x})) \geq 6+(n-6)+1=n+1 .
$$

Subcase 2.2.2: Suppose that $v, \bar{v} \in V_{1}$. For each $w \in A$, there exist distinct vertices $u, z \in V(G)$ such that $u, \bar{z} \in V_{2}, u w \in E(G)$ and $\overline{w z} \in E(\bar{G})$. Again, for each $u \in$ $V_{2} \cap V(G)$, since $\operatorname{deg}_{G}(u) \leq 3$, there can only be at most two vertices $a, b \in A$ for which $u a, u b \in E(G)$. Using similar arguments, if $|A| \leq 2$, then $\omega_{G \bar{G}}(f) \geq n+1$. To proceed, we only have to consider the case where $3 \leq|A| \leq 4$. Other cases follow inductively. 
Suppose that $A=\{x, y, w\}$. The only nontrivial scenario is the following: There exist $a, c \in V_{2} \cap V(G)$ and $b \in V(G)$ such that $\bar{b} \in V_{2}, a c \notin E(G), w c \in E(G),\{x, y\} \subseteq N_{G}(a)$, and $\{\bar{x}, \bar{y}, \bar{w}\} \subseteq N_{\bar{G}}(\bar{b})$. Since $\bar{a} \bar{b} \in E(\bar{G}), f(\bar{a})=1$. Thus,

$$
\begin{aligned}
\omega_{G \bar{G}}(f) & =\sum_{u \in\{a, x, y, b, w, c\}}(f(u)+f(\bar{u}))+\sum_{u \in V(G) \backslash\{a, b, c, x, y, w\}}(f(u)+f(\bar{u})) \\
& \geq 7+(n-7)+2 \\
& >n+1 .
\end{aligned}
$$

Finally, suppose that $A=\{x, y, z, w\}$. It is enough to consider only the following nontrivial case: There exist $a, c \in V_{2} \cap V(G)$ and $b \in V(G)$ such that $\bar{b} \in V_{2}, a c \notin E(G),\{x, y\} \subseteq$ $N_{G}(a),\{w, z\} \subseteq N_{G}(c)$, and $\{\bar{x}, \bar{y}, \bar{z}, \bar{w}\} \subseteq N_{\bar{G}}(\bar{b})$. Since $\bar{a} \bar{b}, \bar{c} \bar{b} \in E(\bar{G}), f(\bar{a})=f(\bar{c})=1$. Hence,

$$
\begin{aligned}
\omega_{G \bar{G}}(f) & =\sum_{u \in\{a, b, c, x, y, w, z\}}(f(u)+f(\bar{u}))+\sum_{u \in V(G) \backslash\{a, b, c, x, y, w, z\}}(f(u)+f(\bar{u})) \\
& \geq 8+(n-8)+2 \\
& >n+1 .
\end{aligned}
$$

All of the above cases show that $\gamma_{R}^{P}(G)=\omega_{G \bar{G}}(f) \geq n+1$.

Next, suppose that $\bar{G}$ is the union of $K_{j} \in\left\{K_{1}, K_{2}\right\}$, and let $f=\left(V_{0}, V_{1}, V_{2}\right)$ be a $\gamma_{R}^{P}$-function of $G \bar{G}$. As shown previously, we may assume that $V_{2} \cap V(G) \neq \varnothing$, and if $V_{2}$ contains a dominating vertex of $G$, then $\omega_{G \bar{G}}(f) \geq n+1$. Henceforth, we assume that $V_{2} \cap \operatorname{Dom}(G)=\varnothing$. Pick $v \in \operatorname{Dom}(G)$. Then $\bar{v} \in I s o(\bar{G})$. Note that for all $\bar{x} \in I s o(\bar{G}), x \notin$ $A=\{w \in V(G): f(w)=f(\bar{w})=0\}$ so that $(f(x)+f(\bar{x})) \geq 1$. Also, for all $x, y \in V(G)$ for which $\overline{x y} \in E(\bar{G})$, if $x \in A$, then $\bar{y} \in V_{2}$ and so $(f(x)+f(\bar{x}))+(f(y)+f(\bar{y})) \geq 2$. Thus, if $v \in V_{0}$ and $u \in V(G)$ such that $V_{2} \cap V(G)=\{u\}$, then

$$
\begin{aligned}
\omega_{G \bar{G}}(f)= & (f(u)+f(\bar{u}))+\sum_{\bar{x} \in I s o(\bar{G})}(f(x)+f(\bar{x}))+ \\
& \sum_{\substack{x y \in E(\bar{G}) \\
\geq}}((f(x)+f(\bar{x}))+(f(y)+f(\bar{y}))) \\
& n+1 .
\end{aligned}
$$

On the other hand, if $v \in V_{1}$, then $f(\bar{v})=1$ and

$$
\begin{aligned}
\omega_{G \bar{G}}(f)= & (f(v)+f(\bar{v}))+\sum_{\bar{x} \in I s o(\bar{G}) \backslash\{\bar{v}\}}(f(x)+f(\bar{x}))+ \\
& \sum_{\substack{x y \in E(\bar{G}) \\
\geq}}((f(x)+f(\bar{x}))+(f(y)+f(\bar{y}))) \\
\geq &
\end{aligned}
$$

Therefore, $\gamma_{R}^{P}(G \bar{G}) \geq n+1$. 
As shown by the graph $G$ in Figure 1, strict inequality may be attained in Proposition $2.13(i v)$ if we remove the condition that $\operatorname{deg}_{G}(v) \leq 3$ for all nondominating vertices $v$ of $G$. For such $G, \gamma_{R}^{P}(G \bar{G})=6<|V(G)|+1$.

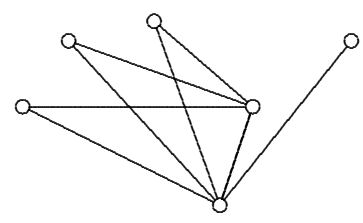

$G$ :

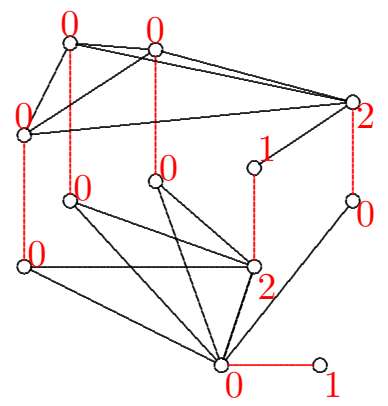

$G \bar{G}$

Figure 1: Graph $G$ with $\gamma(G)=1$ and $\gamma_{R}^{P}(G \bar{G})<|V(G)|+1$

Pick $G=K_{n}$. By Proposition 2.13(iv) and Corollary 2.5,

$$
\gamma_{R}^{P}(G \bar{G})=1+\max \left\{\gamma_{R}^{P}(G), \gamma_{R}^{P}(\bar{G})\right\} .
$$

Observe also that if $v \in V(G)$, then $f=(V(G) \backslash\{v\}, \varnothing,\{v\}) \in P R D(G)$ and $\gamma_{R}^{P}(G \bar{G})=$ $\omega_{G}(f)+n-\left|V_{2}\right|$. The following result shows that these two expressions serve as sharp lower and upper bounds, respectively, of $\gamma_{R}^{P}(G \bar{G})$ for a general graph $G$.

Theorem 2.14. For any graph $G$,

$$
1+\max \left\{\gamma_{R}^{P}(G), \gamma_{R}^{P}(\bar{G})\right\} \leq \gamma_{R}^{P}(G \bar{G}) \leq \rho,
$$

where $\rho=\min \left\{\omega_{G}(f)+n-\left|V_{2}\right|: f=\left(V_{0}, V_{1}, V_{2}\right) \in P R D(G) \cup P R D(\bar{G})\right\}$.

Proof: WLOG assume that for some $f=\left(V_{0}, V_{1}, V_{2}\right)$ on $G, \rho=\omega_{G}(f)+n-\left|V_{2}\right|$. Extend $f$ to $G \bar{G}$ by defining $f(\bar{v})=0$ for all $v \in V_{2}$ and $f(\bar{v})=1$ for all $v \in V(G) \backslash V_{2}$. Then the extension $f \in P R D(G \bar{G})$ and $\gamma_{R}^{P}(G \bar{G}) \leq \omega_{G}(f)+n-\left|V_{2}\right|$. Thus, $\gamma_{R}^{P}(G \bar{G}) \leq \rho$.

In view of Proposition 2.13(iv), we assume that neither $G$ nor $\bar{G}$ is a complete graph. WLOG, assume that $\gamma_{R}^{P}(G) \geq \gamma_{R}^{P}(\bar{G})$. Let $f=\left(V_{0}, V_{1}, V_{2}\right)$ be a $\gamma_{R}^{P}$-function on $G \bar{G}$. If $V(\bar{G}) \subseteq V_{0}$, then $V_{2}=V(G)$ so that $\gamma_{R}^{P}(G \bar{G})=2\left|V_{2}\right|=|V(G \bar{G})|$. Since $G \bar{G}$ is connected, $n=1$ by Corollary 2.5 and Corollary 2.3(ii). This is contradictory to our assumption. Thus, $V(\bar{G}) \cap\left(V_{1} \cup V_{2}\right) \neq \varnothing$. If $V_{2} \cap V(\bar{G})=\varnothing$, then $g=\left(V_{0} \cap V(G), V_{1} \cap V(G), V_{2}\right) \in$ $P R D(G)$. Since $V(\bar{G}) \cap V_{1} \neq \varnothing$,

$$
\gamma_{R}^{P}(G \bar{G})=\omega_{G \bar{G}}(f) \geq \omega_{G}(g)+1 \geq \gamma_{R}^{P}(G)+1 .
$$

Suppose that $V_{2} \cap V(\bar{G}) \neq \varnothing$, and let $A=\left\{v \in V_{0}: V_{2} \cap N_{G \bar{G}}(v)=\{\bar{v}\}\right\}$. Define $g=\left(V_{0}^{*}, V_{1}^{*}, V_{2}^{*}\right)$ on $G$ by

$$
g(x)= \begin{cases}f(x), & \text { if } x \in V(G) \backslash A \\ 1, & \text { if } x \in A .\end{cases}
$$


Then $g \in P R D(G)$ with $V_{0}^{*}=\left(V_{0} \backslash A\right) \cap V(G), V_{1}^{*}=A \cup\left(V_{1} \cap V(G)\right)$ and $V_{2}^{*}=V_{2} \cap V(G)$. Since $\{\bar{v}: v \in A\} \subseteq V_{2} \cap V(\bar{G})$,

$$
\gamma_{R}^{P}(G \bar{G})=\omega_{G}(g)+\sum_{x \in V(\bar{G})} f(x)-|A| \geq \omega_{G}(g)+1 \geq \gamma_{R}^{P}(G)+1
$$

If $G=C_{5}$, then $G$ and $\bar{G}$ are isomorphic and $G \bar{G}$ is isomorphic to the Petersen graph. Observe that $\gamma_{R}^{P}(G \bar{G})=7, \gamma_{R}^{P}(G)=\gamma_{R}^{P}(\bar{G})=4$ and $\rho=8$ so that

$$
1+\max \left\{\gamma_{R}^{P}(G), \gamma_{R}^{P}(\bar{G})\right\}<\gamma_{R}^{P}(G \bar{G})<\rho .
$$

This shows that strict inequality can be attained at each side of the inequalities in Theorem 2.14 .

\subsection{On the edge corona of graphs}

Given graphs $G$ and $H$, we write $H^{u v}$ to denote that copy of $H$ that is being joined with the endvertices of the edge $u v \in E(G)$ in the edge corona $G \diamond H$. If $H=\{x\}$, then we write $V\left(H^{u v}\right)=\left\{x^{u v}\right\}$.

For an $f \in P R D(G)$, we write for each $a, b \in\{0,1,2\}$,

$$
E_{a b}(f ; G)=\{u v \in E(G):(f(u)=a \wedge f(v)=b) \vee(f(u)=b \wedge f(v)=a)\},
$$

where " $\wedge$ " and " $\vee$ " denote "and" and "or", respectively.

Theorem 2.15. Let $G$ be a nontrivial connected graph and $H$ any graph of order $n$. Then

$$
\gamma_{R}^{P}(G \diamond H) \leq \alpha
$$

where

$$
\alpha=\min _{g \in P R D(G)}\left(\omega_{G}(g)+\left|E_{11}(g ; G)\right| \gamma_{R}^{P}(H)+n\left(\left|E_{01}(g ; G)\right|+\left|E_{22}(g ; G)\right|+E_{00}(g ; G) \mid\right)\right),
$$

and this upper bound is sharp.

Proof: Let $g \in P R D(G)$. If no confusion arises, we write $E_{a b}=E_{a b}(g ; G)$. Let $h \in$ $P R D(H)$. For each $a b \in E(G)$, we define a copy $h^{a b}$ of $h$ on $H^{a b}$. Define the function $f=\left(V_{0}, V_{1}, V_{2}\right)$ on $G \diamond H$ by

$$
f(x)= \begin{cases}g(x), & \text { if } x \in V(G) ; \\ h^{u v}(x), & \text { if } x \in V\left(H^{u v}\right), \text { where } u v \in E_{11} \\ 0, & \text { if } x \in V\left(H^{u v}\right), \text { where } u v \in E_{02} \cup E_{12} ; \\ 1, & \text { if } x \in V\left(H^{u v}\right), \text { where } u v \in E_{01} \cup E_{00} \cup E_{22} .\end{cases}
$$


We claim that $f \in P R D(G \diamond H)$. First, note that $\left.f\right|_{G}=g=\left(V_{0} \cap V(G), V_{1} \cap V(G), V_{2} \cap\right.$ $V(G))$. Let $x \in V_{0}$. Suppose that $x \in V(G)$. Then $N_{G \diamond H}(x)=N_{G}(x) \cup\left(\cup_{u \in N_{G}(x)} V\left(H^{u x}\right)\right)$. Since $g \in P R D(G),\left|V_{2} \cap N_{G}(x)\right|=1$, say $V_{2} \cap N_{G}(x)=\{z\}$. Let $u \in N_{G}(x)$, and let $y \in V\left(H^{x u}\right)$. If $u \in V_{0} \cup V_{1}$, then $y \in V_{1}$. On the other hand, if $u \in V_{2}$, then $y \in V_{0}$. Thus, $V_{2} \cap V\left(H^{u x}\right)=\varnothing$. Since $u$ is arbitrary, $V_{2} \cap\left(\cup_{u \in N_{G}(x)} V\left(H^{u x}\right)\right)=\varnothing$ and so $V_{2} \cap N_{G \diamond H}(x)=\{z\}$. Suppose that $x \in V\left(H^{u v}\right)$ for some $u v \in E(G)$. Then $N_{G \diamond H}(x)=\{u, v\} \cup N_{H^{u v}}(x)$. Since $f(x)=0, u v \notin E_{00} \cup E_{22} \cup E_{01}$. If $u v \in E_{11}$, then $h^{u v}(x)=0$ and there exists exactly one $y \in V\left(H^{u v}\right)$ such that $x y \in E\left(H^{u v}\right)$ and $f(y)=h^{u v}(y)=2$. In this case, $V_{2} \cap N_{G \diamond H}(x)=V_{2} \cap N_{H^{u v}}(x)=\{y\}$. Suppose that $u v \in E_{02} \cup E_{12}$. Since $V\left(H^{u v}\right) \subseteq V_{0}$, either $V_{2} \cap N_{G \diamond H}(x)=\{u\}$ or $V_{2} \cap N_{G \diamond H}(x)=\{v\}$. Accordingly, $f \in P R D(G \diamond H)$. Therefore,

$$
\begin{aligned}
\gamma_{R}^{P}(G \diamond H) & \leq \omega_{G}(g)+\left|E_{11}\right| \omega_{H}(h)+\sum_{x \in\left\{V\left(H^{u v}\right): u v \in E_{00} \cup E_{01} \cup E_{22}\right\}} f(x) \\
& =\omega_{G}(g)+\left|E_{11}\right| \omega_{H}(h)+n\left(\left|E_{01}\right|+\left|E_{22}\right|+E_{00} \mid\right) .
\end{aligned}
$$

Since $h$ is arbitrary, the desired inequality holds.

Consider the graph $G \diamond P_{3}$ in Figure 2, where $G$ is the caterpillar $c a(2,0,2)$ with the corresponding vertex labelling. The function $g$ on $V(G)$ given by $g(x)=g(z)=2, g(y)=1$ and $g(x)=0$ else is in $\operatorname{PRD}(G)$. Since $E_{00}=E_{01}=E_{22}=E_{00}=\varnothing, \alpha \leq \omega_{G}(g)=5$ so that $\gamma_{R}^{P}\left(G \diamond P_{3}\right) \leq 5$. Now, note that $\{x, z\}$ is the unique $\gamma$-set of $G \diamond P_{3}$. However, $\{x, z\}$

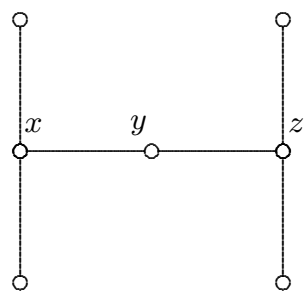

G

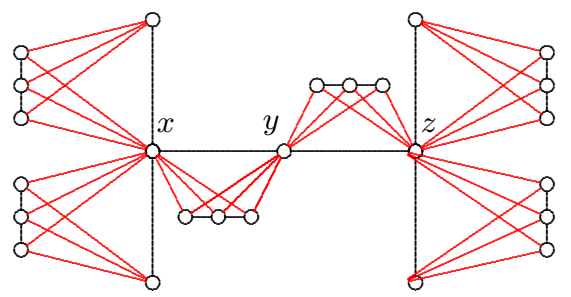

$G \diamond P_{3}$

Figure 2: The edge corona $G \diamond P_{3}$ with $\gamma_{R}^{P}\left(G \diamond P_{3}\right)=5$

does not form the $V_{1} \cup V_{2}$ for any $f=\left(V_{0}, V_{1}, V_{2}\right) \in P R D\left(G \diamond P_{3}\right)$. Thus, $\gamma_{R}^{P}\left(G \diamond P_{3}\right) \geq 5$.

The value of $\alpha$ in Theorem 2.15 is not necessarily determined by a $\gamma_{R}^{P}$-function on $G$. Consider the two copies of the edge corona $P_{5} \diamond C_{4}$ given in Figure 3 with the corresponding assignment of colours to the vertices. Here, we write $P_{5}=\left\{x_{1}, x_{2}, x_{3}, x_{4}, x_{5}\right\}$. Observe that $f=\left(\left\{x_{1}, x_{3}, x_{4}\right\}, \varnothing,\left\{x_{2}, x_{5}\right\}\right)$ is a $\gamma_{R}^{P}$-function on $P_{5}$ (see right-hand side figure), while $g=\left(\left\{x_{1}, x_{5}\right\},\left\{x_{3}\right\},\left\{x_{2}, x_{4}\right\}\right) \in P R D\left(P_{5}\right)$ but not a $\gamma_{R}^{P}$-function on $P_{5}$ (see left-hand side figure). Verify that $\gamma_{R}^{P}\left(P_{5} \diamond C_{4}\right)=5$ and is determined by the function $g$.

From Theorem 2.15 and as illustrated in the preceding example, the value of $\alpha$ in Theorem 2.15 is determined by the functions $g \in P R D(G)$ for which most of the sets 

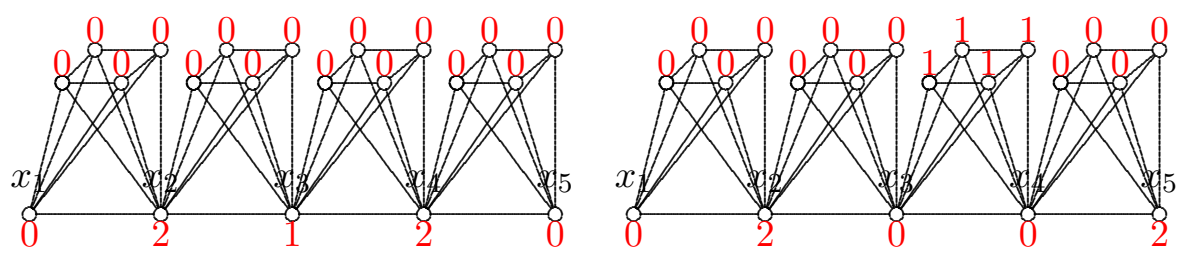

Figure 3: The edge corona $P_{5} \diamond C_{4}$

$E_{00}(g ; G), E_{22}(g ; G), E_{11}(g ; G)$ and $E_{01}(g ; G)$ are empty. In view of such, the following observation can be easily verified.

Corollary 2.16. Let $H$ be any nontrivial graph of order $m$. then

(i) For the path $P_{n}$ on $n \geq 2$ vertices, $\gamma_{R}^{P}\left(P_{n} \diamond H\right)=3\left\lfloor\frac{n-2}{2}\right\rfloor+2$.

(ii) If $m \geq 3$, then for the cycle $C_{n}$ on $n \geq 3$ vertices,

$$
\gamma_{R}^{P}\left(C_{n} \diamond H\right)= \begin{cases}3 k, & \text { if } n=2 k ; \\ 3 k+1+\gamma_{R}^{P}(H), & \text { if } n=2 k+1 .\end{cases}
$$

(iii) If $m \geq 3$, then for $2 \leq n \leq k, \gamma_{R}^{P}\left(K_{n, k} \diamond H\right)=2 n+k$.

Theorem 2.17. Let $G$ be a nontrivial connected graph. Then

$$
\gamma_{R}^{P}\left(G \diamond K_{1}\right)=\min _{g \in P R D(G)}\left(\omega_{G}(G)+\left|E_{00}(g ; G)\right|+\left|E_{01}(g ; G)\right|+\left|E_{11}(g ; G)\right|+\left|E_{22}(g ; G)\right|\right) .
$$

Proof: Put

$$
\alpha=\min \left\{\omega_{G}(G)+\left|E_{00}(g ; G)\right|+\left|E_{01}(g ; G)\right|+\left|E_{11}(g ; G)\right|+\left|E_{22}(g ; G)\right|: g \in P R D(G)\right\} .
$$

By Theorem 2.15, $\gamma_{R}^{P}\left(G \diamond K_{1}\right) \leq \alpha$.

Let $f=\left(V_{0}, V_{1}, V_{2}\right)$ be a $\gamma_{R}^{P}$-function on $G \diamond K_{1}$. Suppose that the restriction $\left.f\right|_{G}$ of $f$ to $G$ is not a perfect Roman dominating function on $G$. We will construct a $\gamma_{R}^{P}$-function $g$ on $G \diamond K_{1}$ such that $\omega_{G \diamond K_{1}}(g)=\omega_{G \diamond K_{1}}(f)$ and its restriction $\left.g\right|_{G}$ to $G$ is a perfect Roman dominating function on $G$. There exists $u \in V_{0} \cap V(G)$ such that $u v \notin E(G)$ for all $v \in V_{2} \cap V(G)$. This means that there exists $v \in N_{G}(u)$ such that $V_{2} \cap N_{G \diamond K_{1}}(u)=\left\{x^{u v}\right\}$.

Case 1: Suppose that $v \notin V_{0}$. Define $f^{1}=\left(V_{0}^{1}, V_{1}^{1}, V_{2}^{1}\right)$ on $G \diamond K_{1}$ by $f^{1}(u)=f^{1}\left(x^{u v}\right)=1$ and $f^{1}(x)=f(x)$ for all $x \in V\left(G \diamond K_{1}\right) \backslash\left\{u, x^{u v}\right\}$. Then $f^{1} \in P R D\left(G \diamond K_{1}\right)$ with $\omega_{G \diamond K_{1}}\left(f^{1}\right)=\omega_{G \diamond K_{1}}(f)$.

Case 2: Suppose that $v \in V_{0}$. If $\left(N_{G}(v) \backslash\{u\}\right) \cap V_{0}=\varnothing$, then take $f^{1}=\left(V_{0}^{1}, V_{1}^{1}, V_{2}^{1}\right)$ on $G$ given by $f^{1}(v)=2, f^{1}\left(x^{u v}\right)=0$ and $f^{1}(x)=f(x)$ for all $x \in V\left(G \diamond K_{1}\right) \backslash\left\{v, x^{u v}\right\}$. Then $f^{1} \in P R D\left(G \diamond K_{1}\right)$ and $\omega_{G \diamond K_{1}}\left(f^{1}\right)=\omega_{G \diamond K_{1}}(f)$. Suppose that $B=\left(N_{G}(v) \backslash\{u\}\right) \cap V_{0} \neq \varnothing$. 
Necessarily, $x^{v w} \in V_{1}$ for each $w \in B$. In this case, take the function $f^{1}=\left(V_{0}^{1}, V_{1}^{1}, V_{2}^{1}\right)$ on $G \diamond K_{1}$ given by

$$
f^{1}(x)= \begin{cases}2, & \text { if } x=v \\ 0, & \text { if } x \in\left\{x^{u v}, x^{v w}: w \in B\right\} \\ 1, & \text { if } x \in B ; \\ f(x), & \text { if } x \in V\left(G \diamond K_{1}\right) \backslash\left(B \cup\left\{x^{v w}: w \in B\right\}\right) .\end{cases}
$$

Then $f^{1} \in \operatorname{PRD}\left(G \diamond K_{1}\right)$ with $V_{0}^{1}=\left(V_{0} \backslash\{v\}\right) \cup\left\{x^{u v}, x^{v w}: w \in B\right\}$, $V_{1}^{1}=\left(V_{1} \backslash\left\{x^{v w}: w \in B\right\}\right) \cup B$ and $V_{2}^{1}=\left(V_{2} \backslash\left\{x^{u v}\right\}\right) \cup\{v\}$. It is easy to verify that $f^{1} \in P R D\left(G \diamond K_{1}\right)$ and $\omega_{G \diamond K_{1}}\left(f^{1}\right)=\omega_{G \diamond K_{1}}(f)$.

If $\left.f^{1}\right|_{G} \notin P R D(G)$, then we follow the same process and obtain $f^{2} \in P R D\left(G \diamond K_{1}\right)$ with $\omega_{G \diamond K_{1}}\left(f^{2}\right)=\omega_{G \diamond K_{1}}\left(f^{1}\right)=\omega_{G \diamond K_{1}}(f)$. If necessary, we do a finitely many repetitions of the process until we obtain a function $g=f^{k} \in P R D\left(G \diamond K_{1}\right)$ for which $\omega_{G \diamond K_{1}}(g)=\omega_{G \diamond K_{1}}(f)$ and $\left.g\right|_{G} \in P R D(G)$. By the definition of $\alpha, \gamma_{R}^{P}\left(G \diamond K_{1}\right)=\omega_{G \diamond K_{1}}(g) \geq \alpha$.

The value of $\gamma_{R}^{P}\left(G \diamond K_{1}\right)$ in Theorem 2.17 is determined by the functions $g \in P R D(G)$ for which the sets $E_{22}$ and $E_{11}$ are empty. With this observation, it can readily be verified that for $n \geq 1$ and $m \geq 3$,

$$
\gamma_{R}^{P}\left(P_{n} \diamond K_{1}\right)=\left\lfloor\frac{n-1}{3}\right\rfloor+\gamma_{R}^{P}\left(P_{n}\right) \text { and } \gamma_{R}^{P}\left(C_{m} \diamond K_{1}\right)=\left\lceil\frac{n}{3}\right\rceil+\gamma_{R}^{P}\left(C_{m}\right) .
$$

\subsection{On the composition of graphs}

Given $S \subseteq V(G[H])$, we write $S_{G}=\{x \in V(G):(x, y) \in S$ for some $y \in V(H)\}$, which is called the projection of $G$ on $G[H]$.

Proposition 2.18. Let $G$ and $H$ be connected graphs, $G$ noncomplete and $H$ of order $n$ with $\gamma(H)=1$. Then

$$
\gamma_{R}^{P}(G[H]) \leq \alpha
$$

where $\alpha=\min \left\{(n-1)\left(\left|V_{1}\right|+\left|V_{2} \cap N_{G}\left(V_{2}\right)\right|\right)+\omega_{G}(f): f=\left(V_{0}, V_{1}, V_{2}\right) \in P R D(G)\right\}$.

Proof: Let $v \in V(H)$ for which $N_{H}[v]=V(H)$. Let $f=\left(V_{0}, V_{1}, V_{2}\right) \in P R D(G)$ such that $V_{2} \neq \varnothing$. Define $g=\left(V_{0}^{*}, V_{1}^{*}, V_{2}^{*}\right)$ on $G[H]$ by

$$
g((x, y))= \begin{cases}0, & \text { if }\left(x \in V_{2} \backslash N_{G}\left(V_{2}\right) \wedge y \neq v\right) \vee\left(x \in V_{0}\right) \\ 1, & \text { if }\left(x \in V_{2} \cap N_{G}\left(V_{2}\right) \wedge y \neq v\right) \vee\left(x \in V_{1}\right) \\ 2, & \text { if } x \in V_{2} \text { and } y=v\end{cases}
$$

with $V_{0}^{*}=\left(\left(V_{2} \backslash N_{G}\left(V_{2}\right)\right) \times(V(H) \backslash\{v\})\right) \cup\left(V_{0} \times V(H)\right), V_{2}^{*}=V_{2} \times\{v\}$ and $V_{1}^{*}=$ $\left(V_{1} \cup V(H)\right) \cup\left(\left(V_{2} \cap N_{G}\left(V_{2}\right)\right) \times(V(H) \backslash\{v\})\right)$. Let $(x, y) \in V_{0}^{*}$. If $x \in V_{2}$, then $x \notin$ $N_{G}\left(V_{2}\right)$ so that $N_{G[H]}((x, y)) \cap V_{2}^{*}=\{(x, v)\}$. If $x \in V_{0}$, then there exists $u \in V_{2}$ such that $N_{G}(x) \cap V_{2}=\{u\}$, which implies that $N_{G[H]}((x, y)) \cap V_{2}^{*}=\{(u, v)\}$. Thus, $g \in$ $\operatorname{PRD}(G[H])$. Therefore, $\gamma_{R}^{P}(G[H]) \leq\left|V_{1}^{*}\right|+2\left|V_{2}^{*}\right|=(n-1)\left(\left|V_{1}\right|+\left|V_{2} \cap N_{G}\left(V_{2}\right)\right|\right)+\omega_{G}(f)$. Since $f$ is arbitrary, the desired inequality is established. 
Proposition 2.19. Let $G$ be a nontrivial connected graph and $p \geq 2$. Then

$$
\gamma_{R}^{P}\left(G\left[K_{p}\right]\right)=\alpha
$$

where $\alpha=\min \left\{(n-1)\left(\left|V_{1}\right|+\left|V_{2} \cap N_{G}\left(V_{2}\right)\right|\right)+\omega_{G}(f): f=\left(V_{0}, V_{1}, V_{2}\right) \in P R D(G)\right\}$.

Proof: Let $f=\left(V_{0}, V_{1}, V_{2}\right)$ be a $\gamma_{P}^{R}$-function on $V(G[H])$. Then $V_{2} \neq \varnothing$ and $V_{0} \neq \varnothing$. First, we claim that $\left(V_{0}\right)_{G} \cap\left(V_{1}\right)_{G}=\varnothing$. Suppose not, and let $(x, y) \in V_{1}$ be such that $(x, z) \in V_{0}$ for some $z \neq y$. There exists unique $(u, v) \in V_{2}$ for which $(x, z)(u, v) \in$ $E\left(G\left[K_{p}\right]\right.$. If $u=x$, then since $y \neq v,(x, y)(u, v) \in E\left(G\left[K_{p}\right]\right)$. Thus, whether $u=x$ or $x \neq u,(x, y)(u, v) \in E\left(G\left[K_{p}\right]\right)$. By Proposition 2.1, there exists $(a, b) \in V_{2} \backslash\{(u, v)\}$ such that $(x, y)(a, b) \in E\left(G\left[K_{p}\right]\right)$. Using the same argument, whether $x=a$ or $x \neq b$, $(x, z)(a, b) \in E\left(G\left[K_{p}\right]\right)$. This is a contradiction since $(x, z) \in V_{0}$.

Fix $v \in V\left(K_{p}\right)$. Define $A=\left\{(x, v): x \in\left(V_{0}\right)_{G} \cap\left(V_{2}\right)_{G}\right\}, B=\left\{(x, y) \in V_{2}: x \notin\left(V_{0}\right)_{G}\right\}$ and $C=\left\{(x, y) \in V_{2}: x \in\left(V_{0}\right)_{G}, y \neq v\right\}$. Put

$$
V_{0}^{*}=\left(V_{0} \backslash A\right) \cup C, \quad V_{1}^{*}=V_{1}, \text { and } V_{2}^{*}=A \cup B .
$$

Then $\left\{V_{0}^{*}, V_{1}^{*}, V_{2}^{*}\right\}$ forms a partition of $V\left(G\left[K_{p}\right]\right)$. Note here that, in particular, since $\left(V_{0}\right)_{G} \cap\left(V_{1}\right)_{G}=\varnothing$ and $V_{1} \cap V_{2}=\varnothing$. Now, let $(x, y) \in V_{0}^{*}$.

Case 1: Suppose that $(x, y) \in V_{0} \backslash A$. There exists $(u, w) \in V_{2}$ such that $N_{G\left[K_{p}\right]}((x, y)) \cap$ $V_{2}=\{(u, w)\}$. If $u \notin\left(V_{0}\right)_{G}$, then $(u, w) \in B$ and $N_{G\left[K_{p}\right]}((x, y)) \cap V_{2}^{*}=\{(u, w)\}$. On the other hand, if $u \in\left(V_{0}\right)_{G}$, then $(u, v) \in A$ and $N_{G\left[K_{p}\right]}((x, y)) \cap V_{2}^{*}=\{(u, v)\}$.

Case 2: Suppose that $(x, y) \in C$ and let $z \in V\left(K_{p}\right) \backslash\{y\}$ for which $(x, z) \in V_{0}$. Since $(x, y)(x, z) \in E\left(G\left[K_{p}\right]\right)$ and $(x, y) \in V_{2}, N_{G\left[K_{p}\right]}((x, z)) \cap V_{2}=\{(x, y)\}$. This means that $(x, w) \notin V_{2}$ for all $w \in V\left(K_{p}\right) \backslash\{y\}$ and $(u, w) \notin V_{2}$ for all $u \in N_{G}(x)$ and for all $w \in V\left(K_{p}\right)$. Thus, $N_{G\left[K_{p}\right]}((x, y)) \cap V_{2}^{*}=N_{G\left[K_{p}\right]}((x, y)) \cap A=\{(x, v)\}$.

Accordingly, the function $g=\left(V_{0}^{*}, V_{1}^{*}, V_{2}^{*}\right) \in P R D\left(G\left[K_{p}\right]\right)$. Since $V_{1}^{*}=V_{1}$ and $\left|V_{2}^{*}\right| \leq$ $\left|V_{2}\right|, \omega_{G\left[K_{p}\right]}(f) \geq \omega_{G\left[K_{p}\right]}(g)$. Because $f$ is a $\gamma_{R}^{P}$-function of $G\left[K_{p}\right], \omega_{G\left[K_{p}\right]}(f)=\omega_{G\left[K_{p}\right]}(g)$ and $g$ is a $\gamma_{R}^{P}$-function of $G\left[K_{p}\right]$.

Define the function $h=\left(V_{0}^{h}, V_{1}^{h}, V_{2}^{h}\right)$ on $G$ by

$$
h(x)= \begin{cases}2, & \text { if } x \in\left(V_{2}^{*}\right)_{G} \\ 1, & \text { if } x \in\left(V_{1}^{*}\right)_{G} \backslash\left(V_{2}^{*}\right)_{G} \\ 0, & \text { else. }\end{cases}
$$

Let $x \in V_{0}^{h}$. Then $(x, y) \in V_{0}^{*}$ for all $y \in V\left(K_{p}\right)$. Pick $y \in V\left(K_{p}\right)$. There exists a unique $(u, v) \in V_{2}^{*}$ for which $(x, y)(u, v) \in E\left(G\left[K_{p}\right]\right)$. It follows that $u \in V_{2}^{h}$ and $u x \in E(G)$. Moreover, $u$ is unique in this sense as $(u, v)$ is for $(x, y)$. Thus, $h \in P R D(G)$.

Finally, let $x, u \in V_{2}^{h}$ for which $x u \in E(G)$. Let $y, v \in V\left(K_{p}\right)$ such that $(x, y),(u, v) \in$ $V_{2}^{*}$. Since $g$ is a $\gamma_{R}^{P}$-function of $G\left[K_{p}\right],(x, a),(u, b) \in V_{1}^{*}$ for all $a \in V\left(K_{p}\right) \backslash\{y\}$ and for all 
$b \in V\left(K_{p}\right) \backslash\{v\}$. On the other hand, by the definition of $h$, for each $x \in V_{1}^{h},(x, y) \in V_{1}^{*}$ for all $y \in V\left(K_{p}\right)$. Thus, $\left|V_{1}^{*}\right| \geq p\left|V_{1}^{h}\right|+(p-1)\left|V_{2}^{h} \cap N_{G}\left(V_{2}^{h}\right)\right|$. Therefore,

$$
\begin{aligned}
\gamma_{R}^{P}\left(G\left[K_{p}\right]\right)=\omega_{G\left[K_{p}\right]}(g) & =\left|V_{1}^{*}\right|+2\left|V_{2}^{*}\right| \\
& \geq p\left|V_{1}^{h}\right|+(p-1)\left|V_{2}^{h} \cap N_{G}\left(V_{2}^{h}\right)\right|+2\left|V_{2}^{h}\right| \\
& =(p-1)\left(\left|V_{1}^{h}\right|+\left|V_{2}^{h} \cap N_{G}\left(V_{2}^{h}\right)\right|\right)+\omega_{G}(h) \\
& \geq \alpha .
\end{aligned}
$$

The desired equality is completed by Proposition 2.18

Equality in Proposition 2.18 is possible even if $H$ is not complete. Consider the graph $G\left[P_{3}\right]$ in Figure 4, with $G$ being the caterpillar graph $c a(0,2,0,2,0)$. Observe that $\alpha=7$.

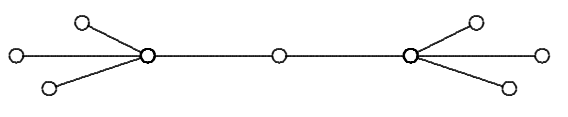

$G$

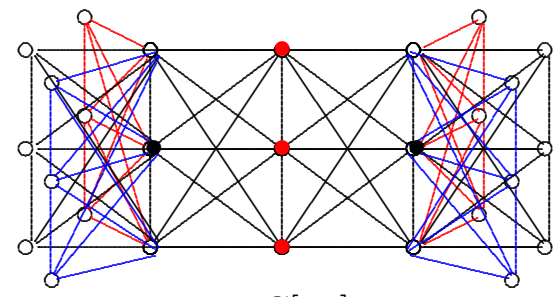

$G\left[P_{3}\right]$

Figure 4: Graph $G$ with $\gamma_{R}^{P}\left(G\left[P_{3}\right]\right)=7$

On the other hand, $\gamma_{R}^{P}\left(G\left[P_{3}\right]\right)=7$, which is determined by $\left(V_{0}, V_{1}, V_{2}\right) \in P R D\left(G\left[P_{3}\right]\right)$, where $V_{1}$ and $V_{2}$ are the sets of all red and all black vertices, respectively, in $G\left[P_{3}\right]$ and $V_{0}=V\left(G\left[P_{3}\right]\right) \backslash\left(V_{1} \cup V_{2}\right)$.

\section{Acknowledgements}

This research is fully funded by the Commission on Higher Education (CHED) Philippines under the CHED K-12 Transition Program and University of Southern Mindanao Research and Faculty Development Program.

\section{References}

[1] H. Abdollhzadeh Ahangar. M. Chellali and S.M. Sheikholeslami, Outer independent double Roman domination. Appl. Math. Comput., 364(124617), 2020.

[2] A. Alhashim. W. Desormeaux and T. Haynes. Roman domination in complementary prisms. Australian Journal of Combinatorics, 68(2):218-228, 2017.

[3] J. Arquilla and H. Fredricksen. "Graphing" an optimal grand strategy. Military Operations Research, 1:3-19, 1995.

[4] C. Berge. The theory of Graphs and its Applications . Wiley, New York, 1962. 
[5] F. Buckley and F. Harary. Distance in Graphs . Addison-Wesley, Redwood City, CA, 1990.

[6] S. Canoy. R. Mollejon and J.G. Canoy. Hop dominating sets in graphs under binary operations. European Journal of Pure and Applied Mathematics, 12(4):1455-1463, 2019.

[7] B. Chaluvaraju and V. Chaitra. Roman domination in complimentary prism. International J.Math. Combi., 2:24-31, 2012.

[8] E. Cockayne. P. Dreyer Jr., S.M. Hedetniemi and S.T. Hedetniemi. Roman domination in graphs. Discrete Mathematics, 278:11-22, 2004.

[9] E. Cockayne and S. Hedetniemi. Towards a theory of domination in graphs. Networks, $7(3): 1977,1977$.

[10] P. Dankelmann, D. Day, D. Erwin, S. Mukwembi, and H. Swart. Domination with exponential decay. Discrete Mathematics, 309:5877-5883, 2009.

[11] W. Desormeaux. T.W. Haynes and M.A. Henning. An extremal problem for total domination stable graphs upon edge removal. Discrete Applied Mathematics, 159:1048-1052, 2011.

[12] O. Favaron. , H. Karami and R. Khoeilar and S.M. Sheikholeslami. On the Roman domination number of a graph. Discrete Mathematics, 309:3447-3451, 2009.

[13] T. Haynes, S.T. Hedetniemi, and P.J. Slater. Fundamentals of Domination in Graphs . Marcel Dekker, Inc., New York, 1998.

[14] M. Henning. W. Klostermeyer and G. MacGillivray. Perfect Roman domination in trees. Discrete Applied Mathematics, 236:235-245, 2018.

[15] M. Henning and W. Klostermeyer. Perfect Roman domination in regular grahs. Applicable Analysis and Discrete Mathematics, 12(1):143-152, 2018.

[16] Y. Kwon and J. Lee. Perfect dominating sets in Cayley graphs. Discrete Applied Mathematics, 162:259-263, 2014.

[17] C.S. Revelle and K.E. Rosing. Defendens imperium romanum: a classical problem in military strategy. Amer. Math. Monthly, 107(7):585-594, 2000.

[18] I. Stewart. Defend the Roman Empire. Sci. Amer., 281(6):136-139, 1999.

[19] J. Yue and J. Song. Note on the perfect Roman domination number of graphs. Applied Mathematics and Computation, 364:1-5, 2020.

[20] J. Yue, M. Wei, M. Li, and G. Liu. On the double roman domination of graphs. Applied Mathematics and Computation, 338:669-675, 2018. 